\title{
Economic Utility of a Blood-Based Genomic Test for the Assessment of Patients with Symptoms Suggestive of Obstructive Coronary Artery Disease
}

\author{
Louis I. Hochheiser, MD, Jessie L. Juusola, PhD, ${ }^{2}$ Mark Monane, MD, MS, ${ }^{2}$ \\ and Joseph A. Ladapo, MD, $\mathrm{PhD}^{3}$
}

\begin{abstract}
Approximately 3 million patients with symptoms suggestive of obstructive coronary artery disease (CAD) present to primary care offices in the United States annually, resulting in approximately $\$ 6.7$ billion in cardiac workup costs. Despite wide application of existing diagnostic technologies, yield of obstructive CAD at invasive coronary angiography (ICA) is low. This study used a decision analysis model to assess the economic utility of a novel gene expression score (GES) for the diagnosis of obstructive CAD. Within a representative commercial health plan's adult membership, current practice for obstructive CAD diagnosis (usual care) was compared to a strategy that incorporates the GES test (GES-directed care). The model projected the number of diagnostic tests and procedures performed, the number of patients receiving medical therapy, type I and type II errors for each strategy of obstructive CAD diagnosis, and the associated costs over a 1-year time horizon. Results demonstrate that GES-directed care to exclude the diagnosis of obstructive CAD prior to myocardial perfusion imaging may yield savings to health plans relative to usual care by reducing utilization of noninvasive and invasive cardiac imaging procedures and increasing diagnostic yield at ICA. At a 50\% capture rate of eligible patients in GES-directed care, it is projected that a commercial health plan will realize savings of $\$ 0.77$ per member per month; savings increase proportionally to the GES capture rate. These findings illustrate the potential value of this new blood-based, molecular diagnostic test for health plans and patients in an age of greater emphasis on personalized medicine. (Population Health Management 2014;17:287-296)
\end{abstract}

\section{Introduction}

$\mathbf{I}_{\mathrm{b}}^{\mathrm{s}}$ N THE United States, approximately 3 million nondiabetic patients present to primary care offices each year with symptoms suggestive of obstructive coronary artery disease (CAD). ${ }^{1-3}$ Many of these patients undergo noninvasive imaging, such as stress myocardial perfusion imaging (MPI) and subsequently are referred to invasive coronary angiography (ICA). However, only approximately $10 \%$ of these patients are diagnosed with stable obstructive CAD as the cause of their symptoms. ${ }^{2,4,5}$ The cardiac evaluation of these patients costs $\$ 6.7$ billion in direct medical costs annually, and the yield of obstructive CAD at ICA is low. ${ }^{6-8}$

The poor disease yield of ICA has raised concerns about the limitations of currently available noninvasive technologies to accurately diagnose obstructive CAD. ${ }^{9}$ Variable performance of noninvasive tests such as stress MPI and stress echocardiography, particularly in relation to factors such as patient obesity, sex, concomitant medication use, and reader interpretation, may contribute to the unnecessary use of ICA in many patients without obstructive CAD. ${ }^{4,10-12}$ A noninvasive diagnostic option with better performance could reduce both the economic burden and adverse clinical consequences of unnecessary cardiac imaging procedures by reducing ICA utilization and the risks of radiation exposure and iatrogenic injury.

With the rapid growth of molecular diagnostics and personalized medicine, genomic diagnostic tests may be able to fill clinical gaps in the care of patients evaluated for obstructive CAD. Recent scientific research has found that multiple genes are activated in circulating blood cells in patients with obstructive CAD. ${ }^{13}$ Accordingly, a new commercially available, validated gene expression score (GES)

\footnotetext{
${ }^{1}$ St John's Medical Center, Jackson, Wyoming.

${ }^{2}$ CardioDx, Inc., Palo Alto, California.

${ }^{3}$ New York University School of Medicine, New York, New York.
} 
diagnostic test has been developed to analyze gene expression for obstructive CAD diagnosis (Corus CAD, CardioDx, Inc., Palo Alto, CA). The GES is a blood-based test that can be administered in the primary care or cardiology setting to assess stable, nondiabetic patients with no history of myocardial infarction (MI) or revascularization presenting with typical or atypical symptoms suggestive of obstructive CAD. As previously described, the GES reports the likelihood that a patient has obstructive CAD, defined as having at least 1 coronary artery with stenosis of $50 \%$ or more as measured by quantitative coronary angiography, on a scale of 1 (low) to 40 (high). ${ }^{14}$ In the Coronary Obstruction Detection by Molecular Personalized Gene Expression (COMPASS) study, its negative predictive value was $96 \%$ for scores of 15 or lower in a patient population that closely resembles the commercial intended use population, in which $\sim 50 \%$ of patients had low GES results. ${ }^{14}$ The clinical utility and safety of the GES also has been described, with physician behavior change based on GES observed in both primary care and cardiology settings. ${ }^{15,16}$

In addition to assessments of the clinical validity and utility of the GES, the economic impact of the test also is an important consideration, particularly for health plans. This study was performed to project the impact of GES-directed care on utilization of cardiac imaging tests and health plan costs for obstructive CAD diagnosis. This analysis aims to help provide guidance for organizations evaluating this novel technology.

\section{Methods}

\section{Overview and model structure}

To assess the economic impact of the new GES test, the study team developed a decision model for the evaluation and treatment of obstructive CAD. The team modeled a population of adults in a representative commercial health plan's membership and compared current practice for obstructive CAD diagnosis (usual care) with a strategy that incorporates the new GES test (GES-directed care). For each strategy, a deterministic decision tree model (in contrast to a stochastic Markov decision tree model $)^{17}$ was used to calculate the number of diagnostic tests and procedures performed, the number of patients receiving medical therapy, and costs associated with diagnosis and treatment of obstructive CAD over a 1-year time horizon. The model was implemented in Microsoft Excel 2011 (Microsoft Corporation, Redmond, WA); the study team previously developed decision analysis models using this software to perform economic evaluations of health care interventions. ${ }^{18-20}$ All costs were assessed from a commercial payer's perspective in 2012 US dollars. One-way sensitivity analyses were conducted on all model parameters by varying each parameter across its range and assessing the impact on results. This method is one of several recommended and widely employed approaches to performing sensitivity analyses in decision analysis models. ${ }^{18-21}$ Table 1 summarizes key model parameters.

The sample health plan membership was assumed to be 500,000 adults based on the average enrollment size of US health plans. ${ }^{22}$ The study team estimated that the incidence of visits to primary care clinicians for stable typical and atypical anginal symptoms in nondiabetic patients with no prior MI or revascularization was $1.04 \% .^{1,3,23}$ Thus, 5200 stable nondiabetic patients with symptoms suggestive of obstructive CAD entered the modeled diagnostic pathways. This patient cohort was assumed to be reflective of the population in the COMPASS study, which was $52 \%$ male with mean age of $56 \pm 10$ years. ${ }^{14}$ The prevalence of obstructive CAD in this patient cohort was $15 \%$, based on the disease prevalence in the COMPASS study. ${ }^{14}$ These demographic parameters were varied in sensitivity analyses to account for variability in member populations regionally and nationally.

\section{Diagnostic strategies and test performance}

Usual care was compared to GES-directed care, where the GES was used prior to MPI to exclude the diagnosis of obstructive CAD. Usual care is defined as a referral from a primary care clinician to a cardiologist for evaluation and subsequent testing using stress MPI. Stress MPI served as the reference test because it is the most frequently used noninvasive imaging test for the assessment of CAD, with over 10 million studies performed annually in the United States. 6,33 Figure 1a illustrates these diagnostic strategies. In sensitivity analyses, assumptions about the percent of patients receiving tests and treatments were varied in the diagnostic strategies.

In the usual care strategy, the study team assumed that all eligible patients presenting to primary care clinicians were referred to a cardiologist and received a stress MPI. Stress MPI test results were normal or abnormal based on obstructive CAD disease prevalence and the test's sensitivity and specificity; MPI's test characteristics were obtained from a meta-analysis. ${ }^{24}$ Patients with normal MPI results received no further cardiac testing but were assumed to receive 1 year of aspirin as a preventive measure. Patients with abnormal MPI results proceeded to ICA, the gold standard for obstructive CAD diagnosis. ${ }^{34}$ The study team assumed that all patients with obstructive CAD at ICA received revascularization, either coronary artery bypass graft surgery or percutaneous coronary intervention, based on rates of revascularization from New York State's Cardiac Diagnostic Catheterization Database. ${ }^{35}$ All patients who proceeded to ICA, regardless of the procedure's findings, were assumed to receive 1 year of optimal medical therapy comprising a statin, beta-blocker, angiotensin-converting enzyme (ACE) inhibitor, and aspirin, based on the Clinical Outcomes Utilizing Revascularization and Aggressive Drug Evaluation (COURAGE) trial regimen. ${ }^{25,36}$

After a health plan introduces GES as a diagnostic option, the portion of patients who will initially receive this test as the first step of their noninvasive cardiac evaluation is unknown. Therefore the study team varied the GES patient capture rate; in the baseline analysis, it was assumed that half of symptomatic patients would receive GES-directed care, with the other half receiving usual care. For patients receiving the GES, the test was performed in the primary care setting. Test results were categorized as low scores $(\leq 15)$ or elevated scores $(>15)$ based on obstructive CAD prevalence and the test's sensitivity and specificity at a threshold of $15 .{ }^{14}$ Because of its negative predictive value of $96 \%$, as described by Thomas et al, ${ }^{14}$ patients with low GES were "ruled-out" for obstructive CAD and therefore received no further cardiac testing. Similar to patients with normal MPI, these patients were assumed to receive 1 year of aspirin. Patients with elevated GES ( $>15$ ) were referred to a cardiologist and received an MPI. Afterward, these 
Table 1. Summary of Key Model Parameters

\begin{tabular}{|c|c|c|c|}
\hline Parameter & Value & Range & Source \\
\hline \multicolumn{4}{|l|}{ Demographic Parameters } \\
\hline Number of eligible patients in plan & 500,000 & $50,000-10,000,000$ & 22 \\
\hline $\begin{array}{l}\text { Annual incidence of nondiabetic } \\
\text { stable chest pain }\end{array}$ & $1.04 \%$ & $0.83-1.25 \%$ & $1,3,23$ \\
\hline Obstructive CAD prevalence & $15 \%$ & $10-25 \%$ & 14 \\
\hline \multirow{2}{*}{\multicolumn{4}{|c|}{$\begin{array}{l}\text { Test Characteristics } \\
\text { MPI }\end{array}$}} \\
\hline & & & \\
\hline Sensitivity & $81 \%$ & $63-93 \%$ & $\begin{array}{l}24 \\
24\end{array}$ \\
\hline $\begin{array}{l}\text { Specificity } \\
\text { GFS }\end{array}$ & $65 \%$ & $10-90 \%$ & 24 \\
\hline \multicolumn{4}{|l|}{ GES at threshold of 15} \\
\hline $\begin{array}{l}\text { Sensitivity } \\
\text { Snecificity }\end{array}$ & $89 \%$ & $78-95 \%$ & 14 \\
\hline Specificity & $52 \%$ & $47-57 \%$ & 14 \\
\hline GES patient capture rate & $50 \%$ & $25-100 \%$ & Assumed \\
\hline $\begin{array}{l}\text { Event Probabilities } \\
\text { Annual probability of nonfatal MI } \\
\text { for false-negative test results }\end{array}$ & $3.7 \%$ & $3.0-4.4 \%$ & 25,26 \\
\hline $\begin{array}{l}\text { Annual probability of receiving diagnosis } \\
\text { for false-negative test results }\end{array}$ & $10.4 \%$ & $8.3-12.5 \%$ & 27 \\
\hline \multicolumn{4}{|l|}{ Cost Parameters (2012 US \$) } \\
\hline \multicolumn{4}{|l|}{ Diagnostic test costs } \\
\hline GES & 1245 & $1000-1500$ & List price \\
\hline MPI & 1276 & $1021-1531$ & Calculated (OptumInsight, Eden Prairie, MN) \\
\hline Invasive coronary angiography & 11,459 & $9167-13,751$ & Calculated (OptumInsight, Eden Prairie, MN) \\
\hline Cardiologist office visit cost & 177 & $142-212$ & 28 \\
\hline \multicolumn{4}{|l|}{ Treatment costs } \\
\hline Preventive therapy (annual supply) ${ }^{\dagger}$ & 0 & $0-300$ & 29-31 \\
\hline Optimal medical therapy for & 1000 & $400-1,200$ & 29-32 \\
\hline
\end{tabular}
obstructive CAD (annual supply)*

21,985 17,588-26,382 Calculated (OptumInsight, Eden Prairie, MN)

CAD, coronary artery disease; GES, gene expression test; MI, myocardial infarction; MPI, myocardial perfusion imaging.

Assumed to be $75-325 \mathrm{mg}$ of aspirin daily.

Assumed to be $40 \mathrm{mg}$ of simvastatin, $100 \mathrm{mg}$ of metoprolol tartrate, $10 \mathrm{mg}$ of lisinopril, and $75-325 \mathrm{mg}$ of aspirin daily.

${ }^{\S}$ Weighted average of percutaneous coronary intervention and coronary artery bypass graft surgery cost based on volume of health claims in database.

patients followed the aforementioned usual care pathway. Test results for MPI were determined by the underlying obstructive CAD prevalence, and prevalence in patients with an elevated GES was higher than in the overall modeled population because many patients without disease were ruled out by the GES prior to MPI.

The model accounted for false-negative test results, or type II errors, for both MPI and GES. Patients with falsenegative results were ascribed a probability of being diagnosed within the 1-year follow-up time frame, either by presenting with new symptoms and subsequently being diagnosed and revascularized, or by presenting with nonfatal MI and being revascularized. These patients were similarly assumed to receive 1 year of optimal medical therapy upon diagnosis. The annual probability of each event was estimated from the literature. ${ }^{25-27}$

\section{Costs}

The study team calculated the costs incurred from a commercial payer perspective for each diagnostic strategy. The model included costs for diagnostic tests (MPI, GES, and ICA), cardiology office visits, revascularization, and medical therapy. Because these costs will vary by payer and geographic region, the team evaluated the impact of varying costs in sensitivity analyses. Per-procedure costs of MPI, ICA, and revascularization were estimated from commercially insured patients with no prior MI or revascularization who underwent cardiac diagnostic testing in a large national de-identified health claims database (OptumInsight, Eden Prairie, MN). The current list price was used for the cost of the GES test. The cost of a cardiologist office visit was estimated from Healthcare Blue Book. ${ }^{28}$ The annual cost of optimal medical therapy for obstructive CAD (daily simvastatin, metoprolol tartrate, lisinopril, and aspirin) was estimated from the 2013 Red Book Online and other sources in the literature. ${ }^{25,29-32,36}$ In the base case it was assumed that payers incurred no cost for preventive therapy of daily aspirin because this is often purchased by patients over the counter; this assumption was varied in sensitivity analyses. (Additional details on cost estimation are available in the Supplementary Data in the online article at www.liebertpub.com/pop).

\section{Results}

\section{Clinical outcomes}

In the usual care diagnostic pathway modeled, all 5200 patients presenting with typical or atypical anginal symptoms were referred to a cardiologist and received a stress 


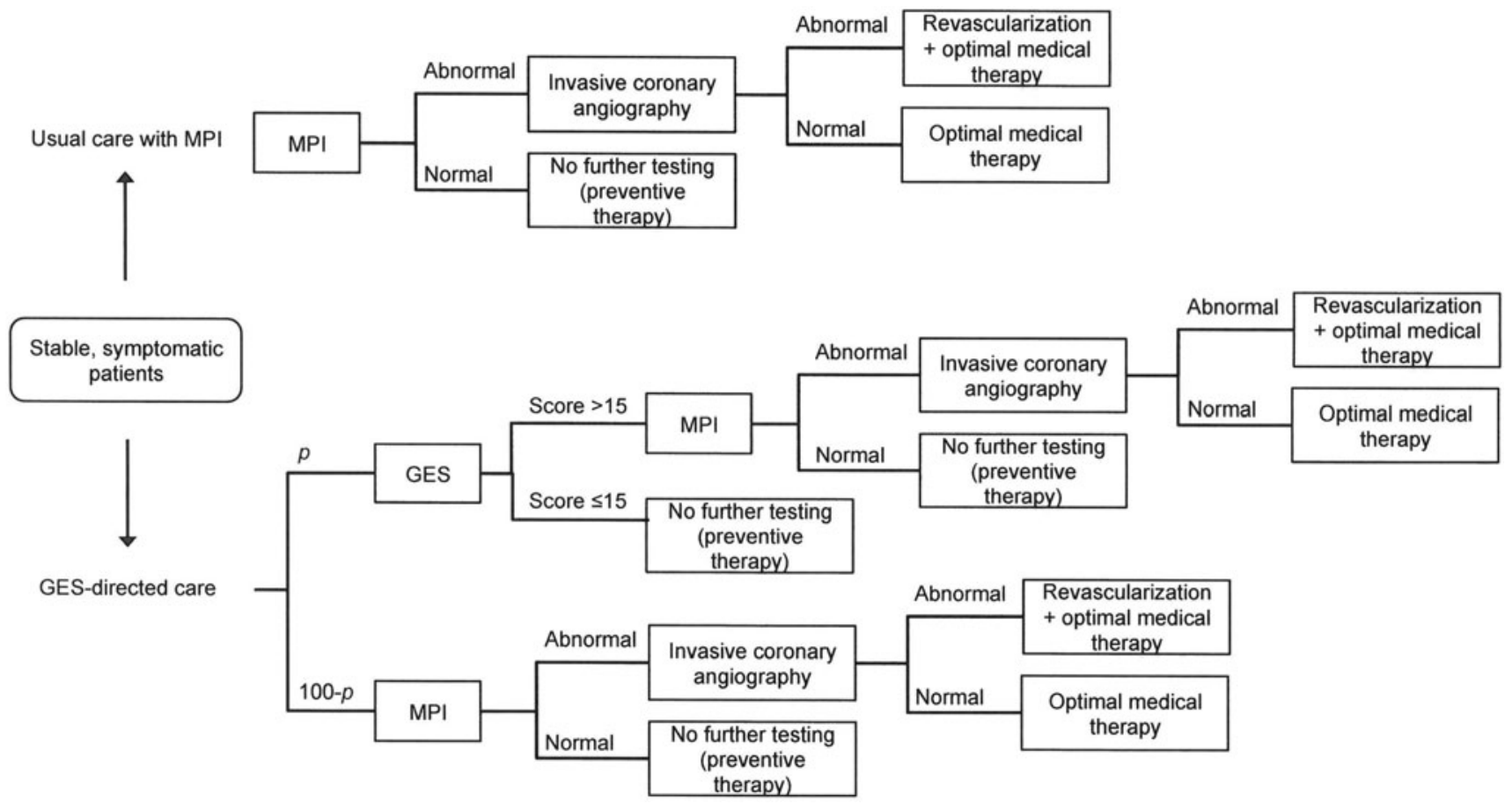

FIG. 1a. Model diagram. The 2 diagnostic strategies examined are illustrated. Patients presenting to primary care settings with stable symptoms suggestive of obstructive CAD progress down each diagnostic pathway based on test results. In the GES-directed care strategy, $P$ represents the patient capture rate. CAD, coronary artery disease; GES, gene expression score; MPI, myocardial perfusion imaging.
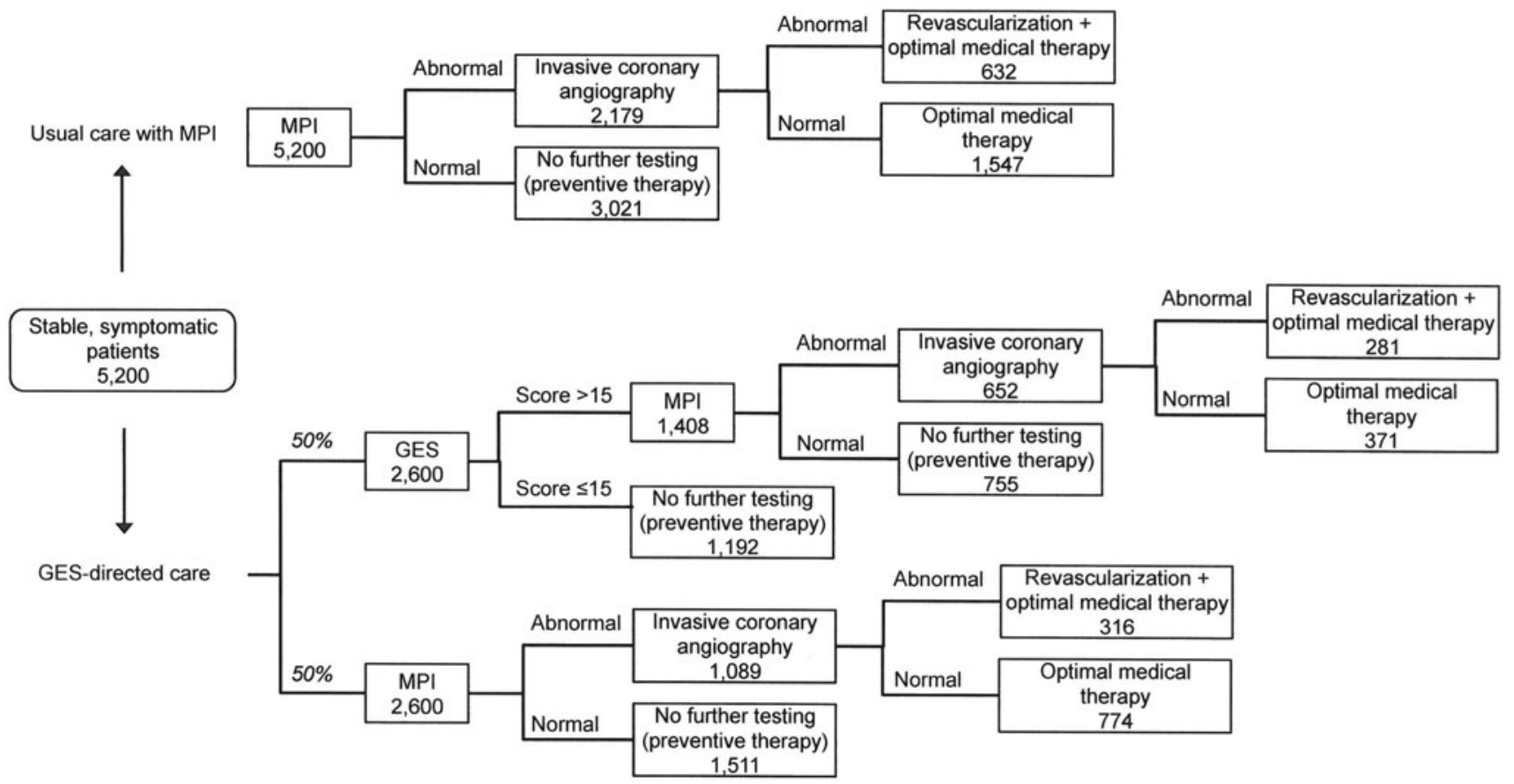

FIG. 1b. Model diagram with patient numbers. The number of patients progressing through each diagnostic pathway based on test results (calculated from test sensitivity, specificity, and prevalence of obstructive CAD) is illustrated for each strategy. The GES patient capture rate in the base case is 50\%. CAD, coronary artery disease; GES, gene expression score; MPI, myocardial perfusion imaging.

MPI. Subsequently, as estimated by the decision tree model and shown in Figure 1b, 2179 (42\%) of those patients underwent ICA because of an abnormal stress MPI result, based on MPI sensitivity and specificity and prevalence of obstructive CAD. Of patients who underwent ICA, only 632
(29\%) were diagnosed with obstructive CAD and revascularized (Figure 2). In comparison, when $50 \%$ of patients received GES-directed care as the initial step in their cardiac workup, the decision tree model estimated that only 4008 patients subsequently received a stress MPI; this 


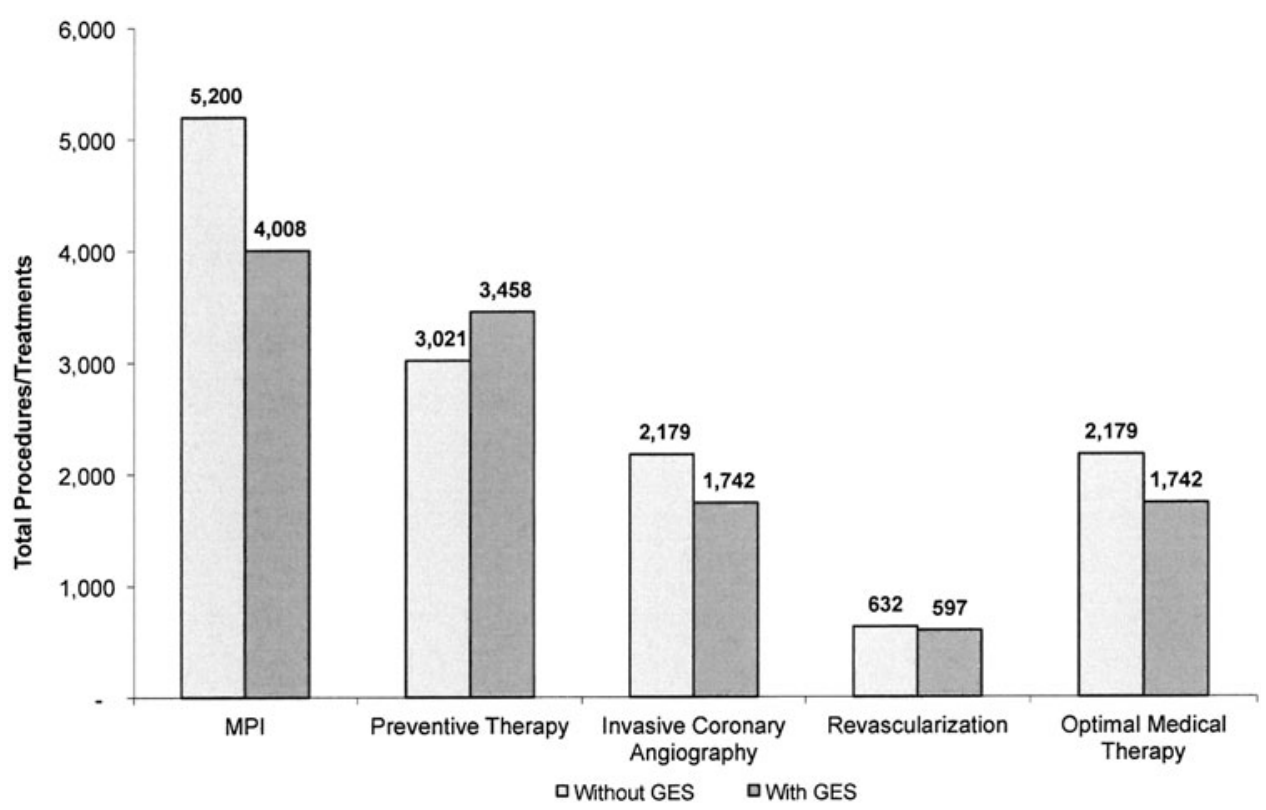

FIG. 2. Effect of GES on number of patients receiving cardiac procedures and treatment. The bars represent the number of patients receiving each test, procedure, or treatment over a 1-year time frame under the 2 strategies: usual care with MPI (Without GES) vs. GES-directed care (With GES). GES, gene expression score; MPI, myocardial perfusion imaging.

represents $1192(23 \%)$ fewer patients compared to usual care (Figure 1b). These 1192 patients received a low GES based on the test's sensitivity and specificity at a score threshold of 15 and prevalence of obstructive CAD in the modeled population, and they were thus "ruled-out" for obstructive CAD. Only 1742 (33\%) patients underwent ICA because of an abnormal stress MPI result, or 437 (20\%) fewer than in usual care, but a similar number of patients were ultimately diagnosed with obstructive CAD and revascularized (Figure $1 \mathrm{~b}$ and Figure 2). Under GES-directed care, a greater number of patients without obstructive CAD were ruled out for a cardiac etiology of their symptoms at an earlier stage in the diagnostic pathway compared to usual care, and fewer cardiac imaging tests were performed.

Ruling patients out for CAD earlier in the diagnostic process also reduced the number of patients receiving extensive medical therapy. In the model, patients who underwent ICA but were not found to have obstructive CAD still received optimal medical therapy of a statin, beta-blocker, $\mathrm{ACE}$ inhibitor, and aspirin. In contrast, those who were ruled out for CAD prior to undergoing ICA received only aspirin. With the GES, the 437 patients who avoided ICA also avoided the more extensive medical regimen and instead received only daily aspirin (Figure 2).

Obstructive disease yield at ICA also was higher under GES-directed care. This signifies a reduction in type I errors, or false positives. With usual care, it was estimated that 632 (29\%) of the 2179 patients undergoing ICA were found to have obstructive $\mathrm{CAD}$, while 1547 patients had false-positive noninvasive imaging results $(35 \%$ of all patients without obstructive CAD), based on MPI specificity (Figure 1b). In contrast, with GES-directed care and a baseline GES patient capture rate of $50 \%$, yield at ICA increased to $34 \%$, and only 1145 patients had false-positive noninvasive testing results ( $26 \%$ of all patients without obstructive CAD). These esti- mates do not include patients with initial false-negative test results who were diagnosed correctly during follow-up.

Although type I errors were reduced under GES-directed care, this pathway also was associated with a modest increase in type II errors, or false negatives, compared to usual care. With usual care, it was estimated that 148 patients had false-negative noninvasive imaging results (19\% of all patients with obstructive CAD) based on MPI sensitivity. With GES-directed care at a $50 \%$ patient capture rate, 183 patients had false-negative test results ( $23 \%$ of all patients with obstructive CAD), either because of a low GES or a normal MPI result. Although the GES test is more sensitive than MPI, the probability of receiving a false-negative test result was higher with a 2-step noninvasive testing pathway than with only 1 noninvasive test.

\section{Budget impact}

GES-directed care was associated with health plan savings, primarily driven by reductions in the number of patients receiving noninvasive and invasive cardiac testing. Under the usual care diagnostic pathway, annual costs to the health plan were \$49.07 million (Table 2). Incorporating a GES-directed care model with a $50 \%$ patient capture rate resulted in a $\$ 4.59$ million $(9.4 \%)$ reduction in costs. Costs for ICA fell by $\$ 5.01$ million $(20.1 \%)$ under GES-directed care, and costs for stress MPI fell by $\$ 1.73$ million $(22.9 \%$ ) (Table 2$)$. These reductions in imaging costs exceeded the $\$ 3.24$ million cost of the GES test itself. Costs for revascularization (which include costs for later diagnosis of false negatives) and optimal medical therapy also fell, but those cost differences were modest relative to savings from fewer ICAs.

One metric often used to evaluate the budget impact of a clinical intervention is per member per month (PMPM) savings. The $9.4 \%$ overall annual reduction in costs from 
Table 2. Model Results-Cost of Strategies for Obstructive CAD Assessment

\begin{tabular}{|c|c|c|c|c|c|c|}
\hline & GES & $M P I^{\dagger}$ & $\begin{array}{c}\text { Invasive Coronary } \\
\text { Angiography }\end{array}$ & $\begin{array}{l}\text { Revascular- } \\
\text { ization }\end{array}$ & $\begin{array}{c}\text { Optimal Medical } \\
\text { Therapy }\end{array}$ & TOTAL \\
\hline Costs with usual care strategy & $\$ 0$ & $\$ 7.56 \mathrm{M}$ & $\$ 24.97 \mathrm{M}$ & $\$ 14.37 \mathrm{M}$ & $\$ 2.18 \mathrm{M}$ & $\$ 49.07 \mathrm{M}$ \\
\hline Costs with GES-directed care strategy & $\$ 3.24 \mathrm{M}$ & $\$ 5.82 \mathrm{M}$ & $\$ 19.96 \mathrm{M}$ & $\$ 13.72 \mathrm{M}$ & $\$ 1.74 \mathrm{M}$ & $\$ 44.48 \mathrm{M}$ \\
\hline Total savings from GES-directed care & $(\$ 3.24 \mathrm{M})$ & $\$ 1.73 \mathrm{M}$ & $\$ 5.01 \mathrm{M}$ & $\$ 0.65 \mathrm{M}$ & $\$ 0.44 \mathrm{M}$ & $\$ 4.59 \mathrm{M}$ \\
\hline$\%$ savings & - & $22.9 \%$ & $20.1 \%$ & $4.5 \%$ & $20.1 \%$ & $9.4 \%$ \\
\hline PMPM savings & $(\$ 0.54)$ & $\$ 0.29$ & $\$ 0.83$ & $\$ 0.11$ & $\$ 0.07$ & $\$ 0.77$ \\
\hline
\end{tabular}

CAD, coronary artery disease; GES, gene expression test; MPI, myocardial perfusion imaging; PMPM, per member per month.

Costs are undiscounted annual costs incurred by a health plan for the assessment of obstructive CAD in nondiabetic patients who present to primary care settings with stable typical or atypical anginal symptoms.

TMPI costs include the cost of a cardiologist office visit along with the cost of the stress MPI itself.

*Revascularization costs include costs from the examined diagnostic strategies as well as procedures in patients who have false-negative test results and are diagnosed during the 1-year follow-up period.

incorporating the GES test in the base case analysis equates to $\$ 0.77$ PMPM savings, driven by $\$ 0.83$ PMPM savings for ICA and \$0.29 PMPM savings for MPI, which outweigh the additional PMPM cost of $\$ 0.54$ for the GES test (Table 2).

\section{Sensitivity analysis}

As previously described, a 1-way sensitivity analysis was conducted for all model parameters. For example, for GES patient capture rate, the study team varied the capture rate from $25 \%$ to $100 \%$ and reestimated the results of the model over that range. In sensitivity analyses, it was found that results were robust to most assumptions, and the GES was associated with PMPM savings for a health plan across all assumptions tested. Results were most substantially affected by MPI specificity and GES patient capture rate, as seen in Figure 3. Other inputs that led to more than a $\$ 0.20$ change in PMPM savings included ICA cost, incidence of stable chest pain, GES sensitivity and specificity, and GES test cost. Although prevalence of obstructive CAD may vary regionally across the United States, results were not sensitive to this input, nor were they sensitive to the assumption that all presenting patients received an MPI in usual care. Results also were insensitive to the cost of optimal medical therapy and revascularization and the percent of patients receiving each of these treatments after ICA. This illustrates the noncritical nature of the assumptions that all ICA patients received optimal medical therapy and all patients with obstructive CAD were revascularized. Similarly, results were insensitive to accounting for a more extensive preventive therapy regimen of a daily statin. (See Supplementary Data available in the online article at www.liebertpub.com/pop.)

The specificity of MPI was a key driver of PMPM savings from GES-directed care because this test characteristic influenced the number of patients receiving false-positive test results and subsequently undergoing an unnecessary ICA, a main source of cost reductions under GES-directed care. Studies of MPI performance have found widely varying estimates of specificity, so the study team tested a similarly wide range of values. ${ }^{24}$ As the specificity of MPI decreased from the base case of $65 \%$, overall PMPM savings from GES-directed care increased because there were more false-positive test results under usual care that were averted by the GES. The opposite was true as the specificity of MPI increased.
The diffusion of GES-directed care in a health plan's population will vary with factors such as physician access to and awareness of the test. In the base case, it was assumed that $50 \%$ of eligible patients would receive GES-directed care. Health plan PMPM savings increased proportionally to the GES capture rate. At $100 \%$ capture rate, the GES led to PMPM savings of $\$ 1.53$ (Figure 4). Only $25 \%$ of patients underwent ICA, compared with $42 \%$ under usual care, and obstructive disease yield at ICA increased to $43 \%$ from $29 \%$ under usual care.

ICA was the most costly procedure whose volume was substantially impacted by GES-directed care. In sensitivity analyses, PMPM savings from GES rose and fell with the cost of ICA. The incidence of office visits for nondiabetic patients presenting with typical or atypical anginal symptoms is also an important input to vary, because PMPM savings depend on the proportion of the health plan's membership affected by the GES. Increasing or decreasing this incidence by $20 \%$ resulted in PMPM savings ranging from $\$ 0.61$ when incidence was minimized, to $\$ 0.92$ when incidence was maximized. As GES sensitivity and specificity were varied within the $95 \%$ confidence intervals reported by Thomas et al, ${ }^{14}$ PMPM savings ranged from $\$ 0.66$ to $\$ 0.96$. PMPM savings also increased as the GES test cost fell. For example, at a GES test cost of $\$ 1050$ (a $15 \%$ reduction from the base case), PMPM savings increased to $\$ 0.85$.

Although stress MPI was used as the reference diagnostic test because of its prominent role in the workup of patients for obstructive CAD, the impact of using coronary computed tomography angiography (CTA) instead also was evaluated. Coronary CTA is a more accurate test diagnostically than MPI, but it is used infrequently in the United States by comparison. ${ }^{33}$ When stress MPI was replaced with coronary CTA, at a coronary CTA cost of $\$ 420$ and sensitivity and specificity of $92 \%$ and $79 \%$, respectively, GES yielded health plan savings, with PMPM savings of \$0.29 at a $50 \%$ GES capture rate. $8,18,37,38$ At $100 \%$ capture rate, PMPM savings increased to $\$ 0.57$.

\section{Discussion}

This analysis demonstrates that the clinical use of a new GES test for assessment of obstructive CAD in symptomatic nondiabetic patients (GES-directed care) may reduce the use of cardiac imaging procedures and yield savings to health 


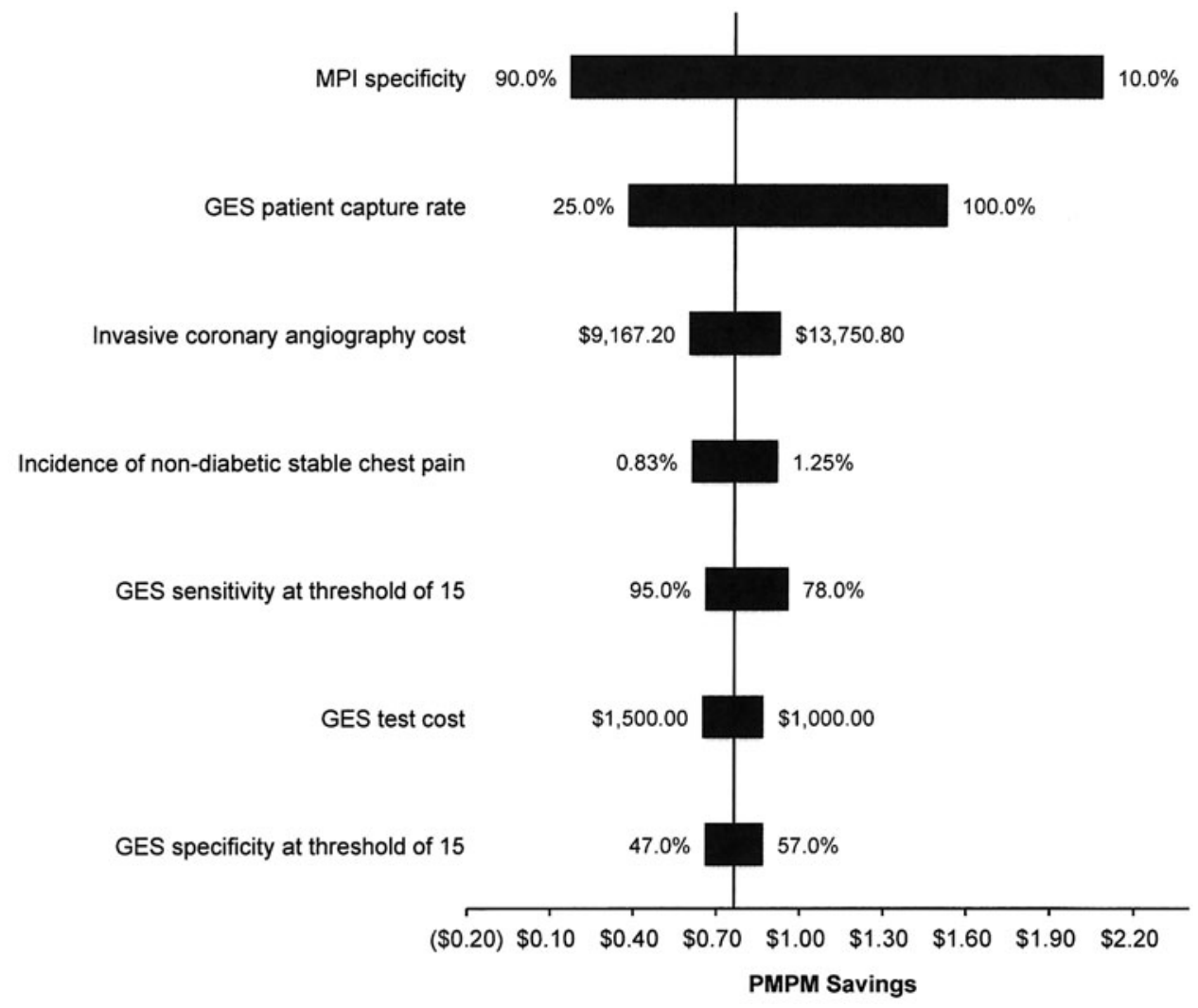

FIG. 3. Tornado diagram. The tornado diagram shows results of univariate sensitivity analysis for the 7 inputs with the largest impact on model results. Overall PMPM savings are displayed along the horizontal axis, and the vertical line indicates the base case result. Ranges tested were based on available literature or were varied $+/-20 \%$ from the base case in the absence of other data. GES, gene expression score; MPI, myocardial perfusion imaging; PMPM, per member per month.

plans relative to usual care by improving diagnostic accuracy in the cardiac workup. It was found that, with only half of eligible patients receiving GES-directed care, a commercial health plan would realize savings of $\$ 0.77$ PMPM, a $9.4 \%$ reduction in total costs, which compares favorably to other budget impact evaluations in common ambulatory conditions. ${ }^{39-41}$ These savings increase in proportion to the GES capture rate. The test reduces costs for health plans by appropriately preventing patients without obstructive CAD from undergoing further cardiac evaluation compared to usual care with MPI alone (ie, reducing the number of false-positive noninvasive test results), thus reducing unnecessary and costly ICA procedures and increasing ICA diagnostic yield.

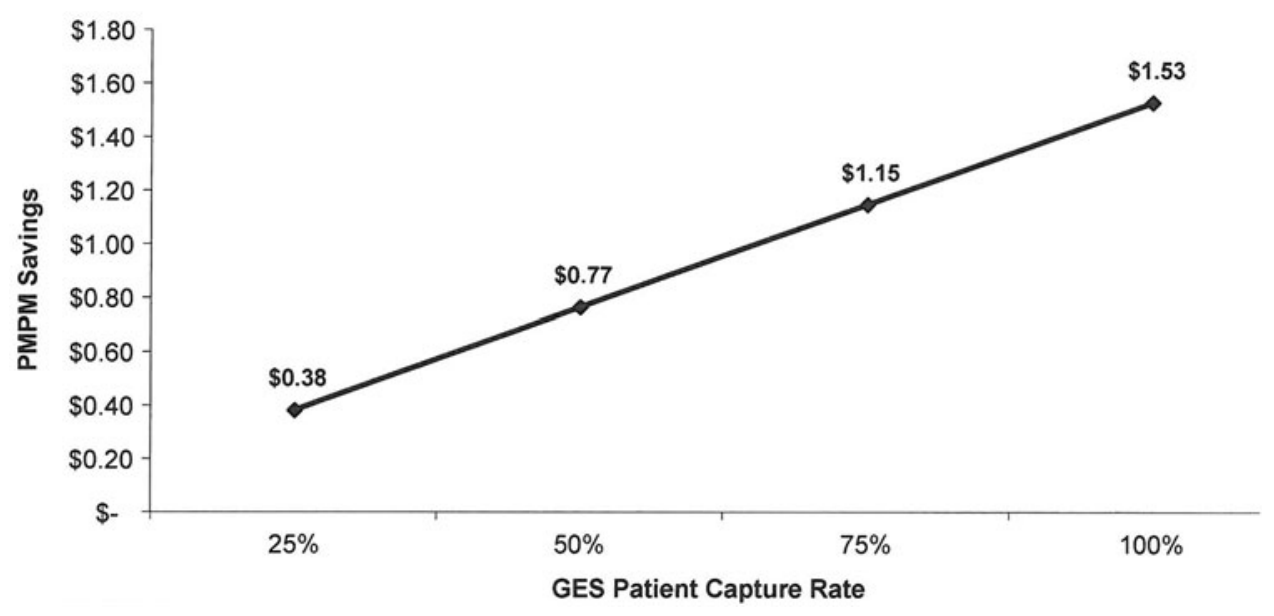

FIG. 4. PMPM savings by GES patient capture rate. Overall PMPM savings from GES-directed care as compared with usual care with stress MPI are plotted by GES patient capture rate. GES, gene expression score; MPI, myocardial perfusion imaging; PMPM, per member per month. 
The reduction in noninvasive and invasive testing under GES-directed care also may yield improvements in the quality of care that patients receive. Stress MPI and ICA expose patients to radiation; stress MPI, in particular, exposes patients to an average effective radiation dose of $15.6 \mathrm{mSv}$ - equivalent to 39 mammograms or almost 800 chest X-rays. ${ }^{42,43}$ In a health plan with 500,000 members, the 1192 stress MPIs averted under GES-directed care at a $50 \%$ patient capture rate equate to a substantial reduction in radiation of approximately $18,600 \mathrm{mSv}$. Furthermore, because ICA is an invasive procedure, it is associated with iatrogenic injury, such as arterial bleeding at the access site, internal bleeding from the catheter puncturing an artery, or stroke or heart attack from atherosclerotic emboli disrupted by manipulation of the catheter within the aorta and coronary arteries. ${ }^{4-46}$ In addition, the contrast agent used during the procedure may cause anaphylaxis, renal impairment, or injury. ${ }^{46}$ Reducing patients' unnecessary exposure to ICA therefore reduces the risk of morbidity and mortality associated with ICA's complications.

A GES for obstructive CAD also may improve patient care by empowering primary care clinicians in the evaluation of patients suspected of having obstructive CAD. Recent initiatives, such as the introduction of patientcentered medical homes as a framework for increasing value in health care, often within accountable care organizations, illustrate the importance of primary care clinicians in quality improvement. ${ }^{47,48}$ As health care's focus shifts toward primary care, primary care clinician access to high-quality, cost-efficient diagnostic tools may improve patient and health plan outcomes. The GES may help meet this need because, as a test that requires only a blood draw at the point of care, it can be readily performed in the primary care setting. In contrast, many advanced cardiac imaging procedures such as stress MPI often require a referral to a cardiologist. Patients with low GES can avoid referrals and their care can continue to be directed by their primary care clinician. Under this scenario, the primary care clinician is better able to evaluate the patient for noncardiac causes of their symptoms in a timelier and more efficient fashion.

The recent trend of hospitals buying or leasing cardiology practices also may have favorable economic implications for use of the GES. ${ }^{49,50}$ Because of these purchase or lease agreements, outpatient imaging tests, such as stress MPI, performed by cardiologists within a hospital-affiliated group can be reimbursed at hospital outpatient rates, which are typically considerably higher than analogous rates for office-based procedures. $^{8,51}$ In settings where health plans are paying higher rates for stress MPI, use of the GES will be comparably more economically favorable.

This study has several limitations. First, simplifying assumptions were made to ensure the analysis was tractable and that the model was transparent. Although this is a limitation of all models, these types of analyses are valuable and have been used to inform clinical guidelines, ${ }^{52-55}$ their wider use also is recommended by the Institute of Medicine. ${ }^{56,57}$ It was assumed that all nondiabetic patients presenting to a primary care setting with symptoms suggestive of obstructive CAD follow a single diagnostic pathway-referral to a cardiologist for stress MPI. Despite the significantly disproportionate use of stress MPI relative to other diagnostic tests for CAD in the United States, in practice there is variability in the utilization of noninvasive imaging tests, the sequence of testing, and the setting of care. However, sensitivity analysis demonstrated that GESdirected care resulted in PMPM savings even when compared to a more accurate and less costly diagnostic modality such as coronary CTA. Another limitation is that the model did not account for costs associated with cancer risk from radiation. Incorporating longer-term consequences from radiation exposure may have led to greater PMPM savings from GES. Similarly, the study team did not account for the cost of complications from ICA. Furthermore, it was assumed that the referral to a cardiologist would be associated with only 1 advanced noninvasive test, but such office visits are often accompanied by additional noninvasive tests as well as repeat office visits. This is an issue that has received recent attention and scrutiny. ${ }^{58}$ Incorporating these factors may have increased the economic savings associated with use of GES. Finally, the analyses were performed from a payer perspective and therefore excluded other socially important costs, such as those associated with productivity losses and patient time spent undergoing noninvasive and invasive imaging procedures.

In conclusion, this study demonstrates that clinical use of the GES test to exclude the diagnosis of obstructive CAD prior to noninvasive cardiac imaging (GES-directed care) may improve upon usual care and reduce the cost of cardiac evaluation in a payer model, with estimated PMPM savings of $\$ 0.77$. The novel characteristics of the bloodbased gene-expression test allow primary care physicians to readily perform the test in the primary care setting and safely exclude obstructive CAD as the cause of patients' symptoms. GES-directed care also may improve diagnostic accuracy and reduce the volume of noninvasive and invasive imaging procedures, with fewer patients undergoing unnecessary ICAs. The study team believes these findings illustrate the potential value of this new molecular diagnostic test for health plans and patients in an age of greater emphasis on personalized medicine.

\section{Author Disclosure Statement}

Drs. Hochheiser, Juusola, Monane, and Ladapo declared the following potential conflicts of interest with respect to the research, authorship, and/or publication of this article: Drs. Juusola and Monane are employees and shareholders of CardioDx, developer of the gene expression score diagnostic test evaluated in this article. Dr. Ladapo has received consulting fees from CardioDx. Dr. Hochheiser is a consultant to CardioDx. The authors received no financial support for the research, authorship, and/or publication of this article.

\section{References}

1. Centers for Disease Control and Prevention. National ambulatory medical care survey: 2010 summary tables. Available at: <http://www.cdc.gov/nchs/data/ahcd/namcs_summary/ 2010_namcs_web_tables.pdf $>$. Accessed July 8, 2013.

2. Cayley WE Jr. Diagnosing the cause of chest pain. Am Fam Physician 2005;72:2012-2021.

3. Woodwell DA, Cherry DK. National ambulatory medical care survey: 2002 summary. Adv Data 2004;346:1-44. 
4. Bosner S, Becker A, Haasenritter J, et al. Chest pain in primary care: epidemiology and pre-work-up probabilities. Eur J Gen Pract 2009;15:141-6.

5. Klinkman MS, Stevens D, Gorenflo DW. Episodes of care for chest pain: a preliminary report from MIRNET. Michigan Research Network. J Fam Pract 1994;38:345-352.

6. IMV. 2011 Nuclear medicine market outlook report. Des Plaines, IL: IMV Medical Information Division, Inc., 2011.

7. IMV. 2008 Cardiac catheterization lab market summary report. Des Plaines, IL: IMV Medical Information Division, Inc., 2008.

8. Centers for Medicare \& Medicaid Services. Physician fee schedule lookup tool. Available at: < http://www.cms.gov/ Medicare/Medicare-Fee-for-Service-Payment/PFSlookup/ index.html >. Accessed March 30, 2012.

9. Brenner DJ. Medical imaging in the 21 st century-getting the best bang for the rad. N Engl J Med 2010;362:943-945.

10. Patel MR, Peterson ED, Dai D, et al. Low diagnostic yield of elective coronary angiography. $N$ Engl J Med 2010;362: 886-895.

11. Carlisle DM, Leape LL, Bickel S, et al. Underuse and overuse of diagnostic testing for coronary artery disease in patients presenting with new-onset chest pain. Am J Med 1999;106:391-398.

12. Shaw LJ, Mieres JH. The role of noninvasive testing in the diagnosis and prognosis of women with suspected CAD. $J$ Fam Pract 2005;suppl:4-5, 7.

13. Elashoff MR, Wingrove JA, Beineke P, et al. Development of a blood-based gene expression algorithm for assessment of obstructive coronary artery disease in non-diabetic patients. BMC Med Genomics 2011;4:26.

14. Thomas GS, Voros S, McPherson JA, et al. A blood-based gene expression test for obstructive coronary artery disease tested in symptomatic nondiabetic patients referred for myocardial perfusion imaging the COMPASS study. Circ Cardiovasc Genet 2013;6:154-162.

15. McPherson JA, Davis K, Yau M, et al. The clinical utility of gene expression testing on the diagnostic evaluation of patients presenting to the cardiologist with symptoms of suspected obstructive coronary artery disease: results from the IMPACT (Investigation of a Molecular Personalized Coronary Gene Expression Test on Cardiology Practice Pattern) trial. Crit Pathw Cardiol 2013;12:37-42.

16. Herman L, Froelich J, Kanelos D, et al. Improved diagnostic testing patterns using a genomic-based personalized medicine test among patients presenting to primary care clinicians with symptoms of suspected obstructive coronary artery disease: results from the IMPACT-PCP (Investigation of a Molecular Personalized Coronary Gene Expression Test on Primary Care Practice Pattern) trial. J Am Board Fam Med In press.

17. Weinstein MC, O'Brien B, Hornberger J, et al. Principles of good practice for decision analytic modeling in health-care evaluation: report of the ISPOR Task Force on Good Research Practices-modeling studies. Value Health 2003;6: 9-17.

18. Ladapo JA, Jaffer FA, Hoffmann U, et al. Clinical outcomes and cost-effectiveness of coronary computed tomography angiography in the evaluation of patients with chest pain. J Am Coll Cardiol 2009;54:2409-2422.

19. Ladapo JA, Jaffer FA, Weinstein MC, Froelicher ES. Projected cost-effectiveness of smoking cessation interventions in patients hospitalized with myocardial infarction. Arch Intern Med 2011;171:39-45.
20. Ladapo JA, Shaffer JA, Fang Y, Ye S, Davidson KW. Costeffectiveness of enhanced depression care after acute coronary syndrome: results from the Coronary Psychosocial Evaluation Studies randomized controlled trial. Arch Intern Med 2012;172:1682-1684.

21. Drummond MF, Sculpher MJ, Torrance GW, O'Brien BJ, Stoddart GL. Methods for the economic evaluation of health care programmes, 3rd ed. New York: Oxford University Press, 2005.

22. AIS's directory of health plans. Washington, DC: Atlantic Information Services, Inc., 2012.

23. An exploratory report of chest pain in primary care. A report from ASPN. J Am Board Fam Pract 1990;3:143-150.

24. Mowatt G, Vale L, Brazzelli M, et al. Systematic review of the effectiveness and cost-effectiveness, and economic evaluation, of myocardial perfusion scintigraphy for the diagnosis and management of angina and myocardial infarction. Health Technol Assess 2004;8:iii-iv, 1-207.

25. Boden WE, O'Rourke RA, Teo KK, et al. Optimal medical therapy with or without PCI for stable coronary disease. $N$ Engl J Med 2007;356:1503-1516.

26. Kuntz KM, Fleischmann KE, Hunink MG, Douglas PS. Cost-effectiveness of diagnostic strategies for patients with chest pain. Ann Intern Med 1999;130:709-718.

27. Metz LD, Beattie M, Hom R, Redberg RF, Grady D, Fleischmann KE. The prognostic value of normal exercise myocardial perfusion imaging and exercise echocardiography: a meta-analysis. J Am Coll Cardiol 2007;49:227-237.

28. Healthcare blue book. CAREOperative, 2013. Available at: $<$ http://www.healthcarebluebook.com/page_Results.aspx?id= $220 \&$ dataset $=$ MD\&g $=$ Office Visit $\% 2 \mathrm{C}$ New Patient $\% 2 \mathrm{C}$ Level 3>. Accessed July 22, 2013.

29. Curtiss FR, Fairman KA. Tough questions about the value of statin therapy for primary prevention: did JUPITER miss the moon? J Manag Care Pharm 2010;16:417-423.

30. Heart Protection Study Collaborative Group. Statin costeffectiveness in the United States for people at different vascular risk levels. Circ Cardiovasc Qual Outcomes 2009;2:65-72.

31. Jick H, Wilson A, Wiggins P, Chamberlin DP. Comparison of prescription drug costs in the United States and the United Kingdom, part 1: statins. Pharmacotherapy 2012; 32:1-6.

32. Truven Health Analytics. Red book online. Available at: $<$ http://www.redbook.com/redbook/online/>. Accessed April 1, 2013.

33. IMV. Present practices and future directions in cardiac imaging: the cardiologists' perspective, 2011-2014. Des Plaines, IL: IMV Medical Information Division, Inc., 2011.

34. Ryan TJ. The coronary angiogram and its seminal contributions to cardiovascular medicine over five decades. Circulation 2002;106:752-756.

35. Hannan EL, Samadashvili Z, Cozzens K, et al. Comparative outcomes for patients who do and do not undergo percutaneous coronary intervention for stable coronary artery disease in New York. Circulation 2012;125:1870-1879.

36. Qaseem A, Fihn SD, Dallas P, et al. Management of stable ischemic heart disease: summary of a clinical practice guideline from the American College of Physicians/ American College of Cardiology Foundation/American Heart Association/American Association for Thoracic Surgery/Preventive Cardiovascular Nurses Association/ Society of Thoracic Surgeons. Ann Intern Med 2012;157: 735-743. 
37. Gueret P, Deux JF, Bonello L, et al. Diagnostic performance of computed tomography coronary angiography (from the Prospective National Multicenter Multivendor EVASCAN study). Am J Cardiol 2013;111:471-478.

38. Miller JM, Rochitte CE, Dewey M, et al. Diagnostic performance of coronary angiography by 64-row CT. $N$ Engl J Med 2008;359:2324-2336.

39. Schwerner H, Mellody T, Goldstein AB, et al. Evaluating the impact of a disease management program for chronic complex conditions at two large northeast health plans using a control group methodology. Dis Manag 2006;9:34-44.

40. Zajac B. Measuring outcomes of a chronic obstructive pulmonary disease management program. Dis Manag 2002;5:9-23.

41. Dunn JD, Cannon E, Mitchell MP, Curtiss FR. Utilization and drug cost outcomes of a step-therapy edit for generic antidepressants in an HMO in an integrated health system. J Manag Care Pharm 2006;12:294-302.

42. Fazel R, Krumholz HM, Wang Y, et al. Exposure to lowdose ionizing radiation from medical imaging procedures. N Engl J Med 2009;361:849-857.

43. Einstein AJ, Moser KW, Thompson RC, Cerqueira MD, Henzlova MJ. Radiation dose to patients from cardiac diagnostic imaging. Circulation 2007;116:1290-1305.

44. Adams DF, Fraser DB, Abrams HL. The complications of coronary arteriography. Circulation 1973;48:609-618.

45. Kennedy JW. Complications associated with cardiac catheterization and angiography. Cathet Cardiovasc Diagn 1982;8:5-11.

46. Lange RA, Hillis LD. Diagnostic cardiac catheterization. Circulation 2003;107:e111-e113.

47. Riley TA, Janosky JE. Moving beyond the medical model to enhance primary care. Popul Health Manag 2012;15:189193.

48. Centers for Medicare \& Medicaid Services. Comprehensive Primary Care Initiative. Available at: < http://innovation .cms.gov/initiatives/comprehensive-primary-care-initiative/ $>$. Accessed August 7, 2013.

49. Ter Maat S. Cardiologists leaving independent practice at rapid pace. Available at: < http://www.amednews.com/ article/20130204/business/130209997/4/ > . Accessed January 6, 2014.

50. Pear R. Medicare panel urges cuts to hospital payments for services doctors offer for less. Available at: < http:// www.nytimes.com/2013/06/15/health/medicare-panel-urgescuts-to-hospital-payments-for-services-doctors-offer-forless.html?_r =0 >. Accessed January 6, 2014.

51. Alvarez \& Marsal Holdings, LLC. A\&M healthcare insights: hospital acquisition of physicians and pricing power. Available at: <http://www.alvarezandmarsal.com/am-healthcare- insights-hospital-acquisition-physicians-and-pricing-power > Accessed August 7, 2013.

52. Mark DB, Berman DS, Budoff MJ, et al. ACCF/ACR/ AHA/NASCI/SAIP/SCAI/SCCT 2010 expert consensus document on coronary computed tomographic angiography: a report of the American College of Cardiology Foundation Task Force on Expert Consensus Documents. $J$ Am Coll Cardiol 2010;55:2663-2699.

53. Fihn SD, Gardin JM, Abrams J, et al. 2012 ACCF/AHA/ ACP/AATS/PCNA/SCAI/STS guideline for the diagnosis and management of patients with stable ischemic heart disease: a report of the American College of Cardiology Foundation/American Heart Association task force on practice guidelines, and the American College of Physicians, American Association for Thoracic Surgery, Preventive Cardiovascular Nurses Association, Society for Cardiovascular Angiography and Interventions, and Society of Thoracic Surgeons. Circulation 2012;126: e354-e471.

54. Mandelblatt JS, Cronin KA, Bailey S, et al. Effects of mammography screening under different screening schedules: model estimates of potential benefits and harms. Ann Intern Med 2009;151:738-747.

55. Chou R, Croswell JM, Dana T, et al. Screening for prostate cancer: a review of the evidence for the U.S. Preventive Services Task Force. Ann Intern Med 2011;155: $762-771$

56. Mandelblatt J, Schechter C, Levy D, Zauber A, Chang Y, Etzioni R. Building better models: if we build them, will policy makers use them? Toward integrating modeling into health care decisions. Med Decis Making 2012;32: 656-659.

57. Institute of Medicine; Committee on Comparative Effectiveness Research Prioritization. Initial national priorities for comparative effectiveness research. Washington, DC: National Academies Press, 2009.

58. Einstein AJ, Weiner SD, Bernheim A, et al. Multiple testing, cumulative radiation dose, and clinical indications in patients undergoing myocardial perfusion imaging. JAMA 2010;304:2137-2144.

Address correspondence to: Joseph A. Ladapo, MD, PhD NYU School of Medicine 277 30th Street, Floor 6, Room 614 New York, NY 10016

E-mail: joseph.ladapo@nyumc.org 